\title{
Modelling the IR spectra of aqueous metal carboxylate complexes: correlation between bonding geometry and stretching mode wavenumber shifts
}

\author{
Catherine. C. R. Sutton, Gabriel da Silva, and George V. Franks*
}

\begin{abstract}
A widely used principle is that shifts in the wavenumber of carboxylate stretching modes upon bonding with a metal centre can be used to infer if the geometry of the bonding is monodentate or bidentate. We have tested this principle with $a b$ initio modelling for aqueous metal carboxylate complexes and have shown that it does indeed hold. Modelling of the bonding of acetate and formate in aqueous solution to a range of cations was used to predict the infrared spectra of the metal-carboxylate complexes, and the wavenumbers of the symmetric and antisymmetric vibrational modes are reported. Furthermore, we have shown that these shifts in wavenumber occur primarily due to how bonding with the metal changes the carboxylate $\mathrm{C}-\mathrm{O}$ bond lengths and $\mathrm{O}-\mathrm{C}-\mathrm{O}$ angle.
\end{abstract}

\section{Introduction}

In their 1980 review of infrared (IR) spectra of acetate and trifluoroacetate complexes Deacon and Phillips proposed guidelines for identifying the type of bonding between a carboxylate anion and a metal containing species. Specifically, they considered the symmetric and antisymmetric vibrations of the carboxylate group, and studied how the difference between the wavenumbers of the symmetric and antisymmetric peaks (a property termed delta, $\Delta$ ) changes upon bonding. ${ }^{[1]}$ The types of bonding are illustrated in Figure 1. They proposed that for monodentate bonding (also known as unidentate bonding), $\Delta$ increases compared to the free anion; for bidentate chelating $\Delta$ decreases; and for bidentate bridging $\Delta$ remains similar to the free anion. ${ }^{[1]}$ There has been further work in recent years and Nakamoto's book Infrared and Raman Spectra of Inorganic and Coordination Compounds provides an overview of this area. ${ }^{[2]}$ It has been observed that carboxylate bond angle and length are related to the wavenumbers of the vibrational peaks, but that there are other factors as well. ${ }^{[1]}$ The guidelines proposed by Deacon and Phillips are useful for understanding the type of bonding for a particular metal carboxylate, however, as there are known exceptions, the guidelines must be applied judiciously. Indeed, Deacon and Phillips urged caution when applying their guidelines to other carboxylates, stating "it would be unwise to extrapolate to more complex carboxylates". Their paper has

[] Dr C. C. R. Sutton, Dr G. da Silva, Prof G. V. Franks

Chemical and Biomolecular Engineering

The University of Melbourne

Parkville, Victoria, 3010, Australia

E-mail: gvfranks@unimelb.edu.au

Supporting information for this article is given via a link at the end of the document. been cited over 3,000 times and while many authors use the guidelines to help discussion ${ }^{[3]}$, there are cases in the literature where the guidelines are taken as an absolute rule. ${ }^{[4]}$ Such guidelines are useful when interpreting experimental IR spectra in cases where carboxylate anions bond to metal containing species in an immense range of fields, including protein structures [3f, 3p, 5], activity of pharmaceuticals ${ }^{[6]}$, fatty acid monolayers ${ }^{[7]}$, the structure of cross linked polymer gels ${ }^{[8]}$, photochemical systems in solar cells ${ }^{[3 m, 3 q, 3 s, 4 c, 9]}$, and control of the morphology of micro- and nanocrystals ${ }^{[3 \mathrm{~d}, 10]}$. Although many of these systems occur in aqueous solution, the guidelines are based on $\mathrm{x}$-ray data from solid state measurements.<smiles></smiles>

(a)<smiles>[M]O[14C]([R])=O</smiles>

(b)<smiles>[M]O[M]1=C([R])C1O[M]</smiles>

(c)<smiles></smiles>

(d)
Figure 1. Bonding geometries and naming conventions for metal carboxylate complexes. (a) Free anion in aqueous solution, where $\mathrm{n}_{1}$ and $\mathrm{n}_{2}$ are approximately equal. (b) Monodentate bonding, where one oxygen atom is bonded to the metal cation; $n_{1}$ is the $\mathrm{C}-\mathrm{O}$ bond length of the unbound oxygen (shorter) and $n_{2}$ is the $\mathrm{C}-\mathrm{O}$ bond length of the oxygen bonded to the metal cation (longer). (c) Bidentate chelating bond, where both oxygen atoms bond to the metal cation, and $\theta$ is the $\mathrm{O}-\mathrm{C}-\mathrm{O}$ bond angle which is more acute than that of the free anion. (d) Bidentate bridging, where one metal cation is bonded to each oxygen atom, (not investigated in this work).

The guidelines of Deacons and Phillips have been widely used and the approach has been quite useful although it has not yet been rigorously validated. This work sets out to investigate the bonding of a range of cations to acetate and formate in aqueous solution using $a b$ initio modelling. Specifically, we investigate how bonding with metal cations affects the IR spectra of carboxylate compounds. There have been ab initio studies of the bonding of carboxylates to metal cations ${ }^{[11]}$ and to metal ${ }^{[12]}$ and metal oxide ${ }^{[13]}$ surfaces for specific systems, in addition to the experimental IR spectra. These simulation results generally do follow the guidelines but there has not been a fundamental investigation to explain why the $\Delta$ values change as they do. It is also noted that many of these studies did not investigate 
aqueous systems, and therefore do not consider the effects of solvation, which are particularly important for ionic species.

\section{Results and Discussion}

The approach taken here is to initially examine the role that bond angle and bond length play in shifting IR spectra of carboxylate compounds. We then relate these findings to calculated IR spectra for these carboxylates when bonded to a range of metal cations. Bidentate bonds are effectively symmetric, as such, varying the bond angle is proposed to simulate (mimic) the influence of bidentate bonding on the energy, geometry and resulting IR spectra of carboxylate anions participating in bidentate bonds. Monodentate bonds result in the carboxylate containing one double $\mathrm{C}=\mathrm{O}$ bond and one single $\mathrm{C}-\mathrm{O}$ bond, as such, varying the bond length of one $\mathrm{C}-\mathrm{O}$ bond while allowing the other to relax is proposed as a mimic of monodentate bonds.

A bidentate bond involves the cation bonding to both of the carboxylate oxygen atoms; accordingly the $\mathrm{C}-\mathrm{O}$ bond lengths are likely to be equivalent to one another, but the $\mathrm{O}-\mathrm{C}-\mathrm{O}$ bond angle will change from that observed in the free anion. The effect of the bidentate bond on the carboxylate is modelled by specifying the carboxylate bond angle over a range of values and allowing the bond lengths to optimize. Vibrational wavenumbers of the structures with the various bond angles are then calculated so as to determine the effect that geometry alone has on the wavenumber of the symmetric vibration of the carboxylate group $\left(v_{\mathrm{s}}\right)$, the wavenumber of the antisymmetric vibration of the carboxylate group $\left(v_{\text {as }}\right)$, and the difference between the two $(\Delta)$. A cartoon of this process is shown in Figure 1 (c). Figure 2 a shows the wavenumbers plotted against bond angle over the range of $115^{\circ}$ to $130^{\circ}$. The general trend in $v_{\mathrm{as}}$ is that it increases with increasing bond angle. The value of $v_{s}$ remains roughly constant below the bond angle of the free, aqueous carboxylate anion and decreases with increasing bond angle. The overall effect is for $\Delta$ to decrease as the O-C-O bond angle becomes more acute. Assuming that bidentate chelating bonds result in smaller carboxylate bond angles than the free solution structure, this result for aqueous complexes is in accordance with the experimental guideline ${ }^{[1]}$ that bidentate bonds result in a smaller $\Delta$. For formate, the values of $v_{\text {as }}$ range from 1498 to $1658 \mathrm{~cm}^{-1}$, while $v_{\mathrm{s}}$ range from 1287 to $1387 \mathrm{~cm}^{-1}$. For acetate, the values of $v_{\text {as }}$ range from 1507 to $1633 \mathrm{~cm}^{-1}$, while $v_{\mathrm{s}}$ range from 1268 to $1483 \mathrm{~cm}^{-1}$. Discontinuities in the acetate $v_{\mathrm{s}}$ plot occur at transitions between the individual vibrational modes that have the highest intensity.

A monodentate bond involves one oxygen atom (in the carboxylate) forming a single bond to a metal atom. This allows the other oxygen atom to form a $\mathrm{C}=\mathrm{O}$ double bond. As such, a mimic for monodentate bonding is proposed where one $\mathrm{C}-\mathrm{O}$ bond length is specified while all other bonds are allowed to relax. Figure $2 \mathrm{~b}$ shows the specified bond length plotted against the wavenumber for formate and acetate, over a range of $1.20 \AA$ to $1.28 \AA$. For both formate and acetate $v_{\text {as }}$ decreases significantly with increasing bond length. However, $v_{s}$ is relatively invariant, increasing slightly to a maximum at just below the bond length of the relaxed free aqueous anion structure, then decreasing at greater bond lengths. For formate, the values of $v_{\text {as }}$ range from 1552 to $1824 \mathrm{~cm}^{-1}$, while $v_{\mathrm{s}}$ range from 1226 to $1394 \mathrm{~cm}^{-1}$. For acetate, the values of $v_{\mathrm{as}}$ range from 1531 to $1838 \mathrm{~cm}^{-1}$, while $v_{\mathrm{s}}$ range from 1304 to $1449 \mathrm{~cm}^{-1} . \Delta$ is at a minimum within $0.01 \AA$ of the relaxed structure for formate as well as acetate. The fact that $\Delta$ increases when the bond lengths vary from those of the global minimum is intuitive as lengthening one $\mathrm{C}-\mathrm{O}$ bond will result in a contraction of the other, with the resultant structure becoming increasingly asymmetric. As such, these models of monodentate bonding in aqueous solution are consistent with the experimental guidelines determined from solid state data. ${ }^{[1]}$
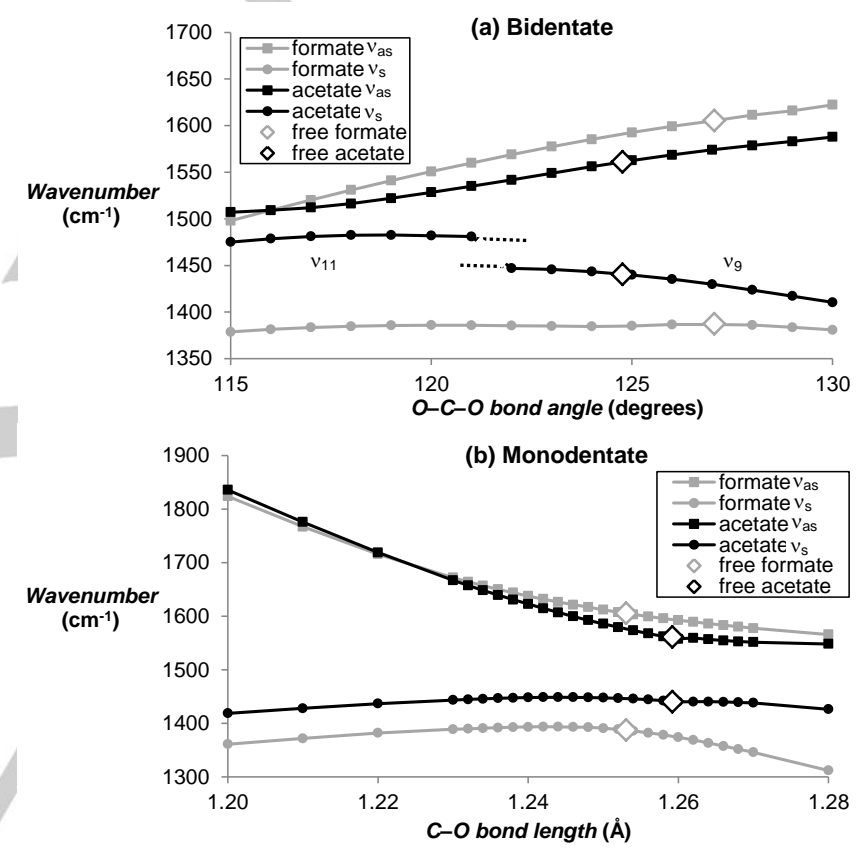

Figure 2. Influence of geometry on wavenumbers of symmetric and antisymmetric vibrational modes of the carboxylate groups of formate and acetate. (a) Bidentate mimic indicating influence of bond angle. (b) Monodentate mimic indicating influence of specified bond length. One individual mode dominated in all cases except for $v_{s}$ of acetate for the bidentate mimic where the highest intensity mode switched from $v_{9}$ to $v_{11}$ at around 121 degrees. The large open symbols are for the free aqueous carboxylates. The black symbols and lines are for acetate and the lighter symbols and lines are for formate. The squares are the antisymmetric modes and the circles are the symmetric modes.

Having seen the effects on $v_{\mathrm{as}}$ and $v_{\mathrm{s}}$ achieved by the mimics, these are now compared to systems where acetate forms complexes with metal cations in aqueous solution. We have studied the $\mathrm{Li}^{+}, \mathrm{Na}^{+}, \mathrm{Mg}^{2+}, \mathrm{Ca}^{2+}, \mathrm{Fe}^{2+}, \mathrm{Cu}^{+}$, and $\mathrm{Zn}^{2+}$ cations, and for each metal carboxylate we identified all stable conformations (see Tables S1 and S2 in Supporting Information). For all complexes bidentate chelating bonding was observed, except for $\mathrm{Cu}^{+}$. In several cases multiple bidentate metal acetate conformers were located, corresponding to rotation of the methyl 
group. For all metals at least one structure corresponding to monodentate bonding was also located, in cis, trans, and/or gauche conformations. The calculated wavenumbers of the antisymmetric and symmetric vibrations are plotted as a function of carboxylate bond lengths and angles in Figure 3 for all stable metal acetates identified. Comparison is also made to the relationships determined for the monodentate and bidentate mimics. This allows for examination of the cause of shifts in wavenumber (i.e., to what extent is the shift captured by changing bond length or angle?). Note that the results for formate showed similar trends, as presented in Figure S1 in the Supporting Information.

The antisymmetric vibrational wavenumbers of the bidentate complexes are plotted against the carboxylate bond angle for acetate in Figure 3a. All bond angles of the cation-carboxylate anion systems are lower than that of the free, aqueous carboxylate anion. The bidentate geometries follow the same trend as the bidentate mimics and $v_{\text {as }}$ decreases with decreasing bond angle, however, the mimic overestimates $v_{\text {as }}$ by up to 20 $\mathrm{cm}^{-1}$. The $v_{\text {as }}$ values for monodentate systems do not follow the prediction of the monodentate mimic as to how this vibrational frequency would change with bond angle, but instead increase with decreasing bond angle (Figure $3 b$ ). This is not surprising as the bond angle is not expected to be influenced significantly by monodentate bonding.

When the metal cations bond to the carboxylate anion, the metal centre influences both carboxylate bond lengths, $n_{1}$ and $n_{2}$. Both bond lengths of all the bidentate systems are greater than those in the aqueous carboxylate anion. The monodentate systems have one C-O bond that is shorter $\left(n_{1}\right)$ and one that is longer $\left(\mathrm{n}_{2}\right)$ than that of the aqueous carboxylate anion. In Figure $3 c$, the antisymmetric vibrational wavenumber is plotted against $\mathrm{n}_{1}$ for the metal cations that form monodentate bonds with acetate. The $v_{\text {as }}$ of monodentate systems with acetate follow the same trend as the monodentate mimics and decrease with bond angle; however the formate mimic (shown in Figure S1 in the Supporting Information) overestimates $v_{\text {as }}$ by around $30 \mathrm{~cm}^{-1}$. In Figure $3 \mathrm{~d}$, one sees that the bidentate geometries also follow the bidentate mimics for bond length and $v_{\text {as }}$ decreases with bond length.

In the Supporting Information (Figures S2 and S3), the symmetric vibrational wavenumber is plotted against bond length and bond angle for acetate and formate. $v_{\mathbf{s}}$ is constant with bond angle for bidentate systems, which is in agreement with the mimic. $v_{s}$ for monodentate systems decrease with decreasing bond angle. The actual decrease is far less than the monodentate mimic predicts. The bidentate metal formate geometries follow the same trend as the bidentate mimics and $v_{\mathrm{s}}$ remains approximately constant with bond length, while the bidentate metal acetate geometries do not trend with bond length. The $v_{s}$ of monodentate systems do increase with bond length, as per the monodentate mimics' predictions; however, the gradient is greater than in the model.
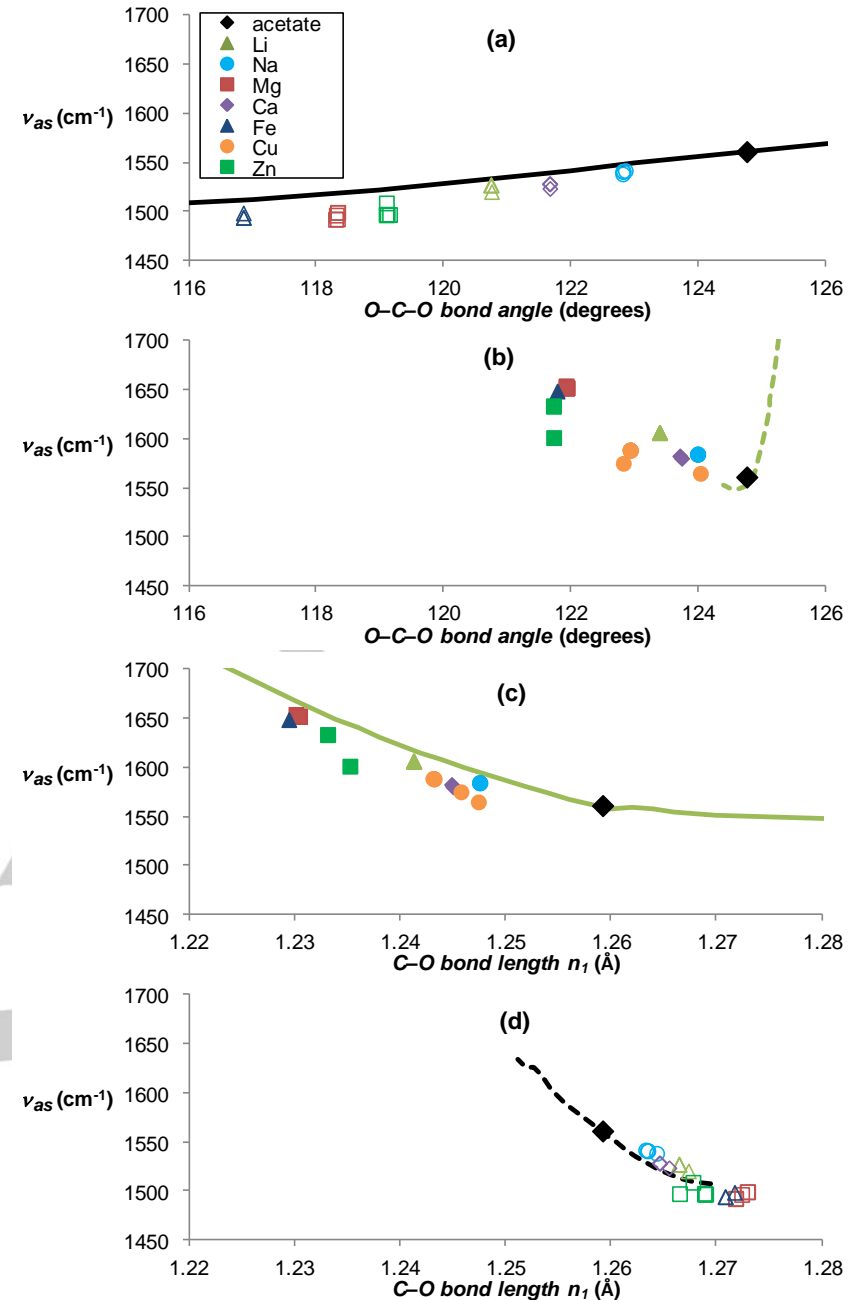

Figure 3. Influence of geometry on antisymmetric vibrational mode wavenumbers of the carboxylate group for acetate. Open dots and black lines are bidentate. Filled dots and lighter colored lines are monodentate. The top two panels show the influence of bond angle where (a) compares the bidentate mimic result (solid line) to the acetate-metal cation bidentate complexes; and where (b) compares the monodentate mimic result (dashed line) to the acetate-metal cation monodentate complexes. The bottom two panels show the influence of bond length where (c) compares the monodentate mimic result (solid line) to the acetate-metal cation monodentate complexes; and where (d) compares the bidentate mimic result (dashed line) to the acetate-metal cation bidentate complexes. Note, the solid lines indicate that the property was specified in the simulation and the dotted lines indicate the property was allowed to relax.

To further investigate the nature of metal carboxylate bonding, $\Delta$ is plotted against the bond energy for acetate in Figure 4. The trend (also observed for formate, shown in Figure S5 in the Supporting Information) is that for bidentate geometries $\Delta$ gets smaller as the magnitude of the bond energy increases, while for monodentate geometries $\Delta$ gets larger as the magnitude of the bond energy increases. The stronger the bonding (of either sort), the greater the deformation and so the greater the influence on $\Delta$; both $v_{\mathrm{as}}$ and $v_{\mathrm{s}}$ change as a function of bond energy (see Figure $\mathrm{S} 6$ in Supporting Information). For bidentate geometries, 
the $v_{\text {as }}$ wavenumber gets lower as the magnitude of the bond energy increases while for monodentate geometries the $v_{\mathrm{as}}$ wavenumber gets higher as magnitude of the bond energy increases. For bidentate geometries the $v_{\mathrm{s}}$ wavenumber does not change significantly, while for monodentate geometries the $v_{\mathrm{s}}$ wavenumber decreases slightly. This reveals that $\Delta$ is dominated by $v_{\text {as }}$. Also of note is that, for any given metal, the bidentate complexes are more stable than the monodentate complexes. This is somewhat surprising as experimental IR spectra of aqueous zinc- and copper-acetate systems lead to greater values of $\Delta$, consistent with monodentate bonding. [3e] However, real metal carboxylate compounds such as these may involve multiple anions and cations (as well as solvent molecules), and we suggest that the treatment of larger structures would be required to fully describe bonding in the metal carboxylates. Future work will examine these possibilities in order to explain what is seen in experimental spectra.

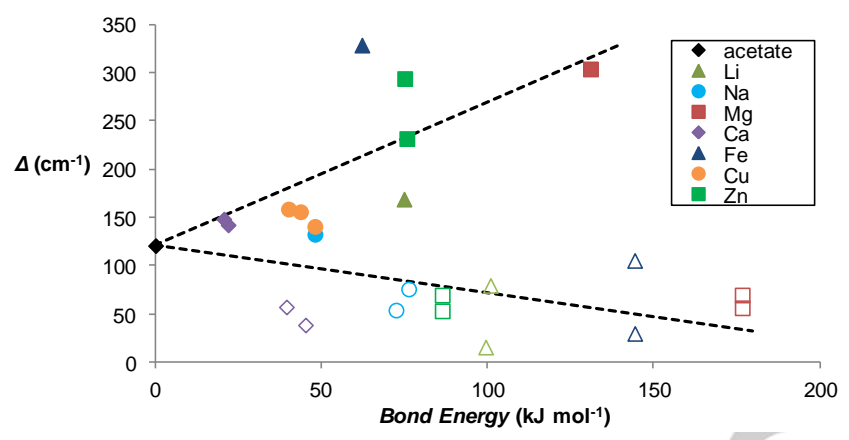

Figure 4. Influence of metal-oxygen bond energy in the metal cation-acetate complexes on delta. Open symbols are bidentate. Filled symbols are monodentate. Dotted lines are lines of best fit passing through $\Delta=121 \mathrm{~cm}^{-1}$ at zero bond energy.

This work has revealed that changes in $v_{\mathrm{s}}$ and $v_{\mathrm{as}}$ (and therefore $\Delta$ ) for a range of metal acetate and formate complexes can be described largely by changes in the $\mathrm{C}-\mathrm{O}$ bond lengths for monodentate structures and by changes in the $\mathrm{O}-\mathrm{C}-\mathrm{O}$ angle for bidentate structures. This provides theoretical validation for the experimental guidelines of Deacon and Phillips. There are, however, remaining differences in calculated $v_{\mathrm{s}}$ and $v_{\mathrm{as}}$ values relative to those inferred purely from the structural mimics, and we now consider what else might contribute to shifts in these vibrational modes. The metal-oxygen bond length does not correlate to changes in the wavenumbers (illustrated in the Supporting Information Figure S4) and so is determined to be unimportant. It can be seen in the Supporting Information (Figures S7 and S8 and related text) that the bidentate mimic successfully predicts the bond lengths of bidentate chelating species relative to each other, but over predicts bond lengths at a given bond angle. The monodentate mimic does not capture the bond angle behavior and under-predicts the length of $n_{2}$. The monodentate mimic has a delocalized electron, however this is not the case for monodentate metal-carboxylate systems; this may account for the differences between the mimics and the metal-carboxylate complexes. This can be quantified by examination of the Mulliken partial charges (Figures S9 and S10). [18] Compared to the metal-carboxylate complexes, the mimics (both monodentate and bidentate) underestimate the value of the partial charge on each of the oxygen atoms as well as the carbon atom.

\section{Conclusions}

The guidelines of Deacon and Phillips, which relate the change in the vibrational modes to the type of bonding occurring, are widely used and have been applied beyond their original scope. This work has provided a theoretical validation of the guidelines for cases of acetate and formate bonding with metals in aqueous solution. A monodentate bond will lead to a greater $\Delta$ (increase in difference in wavenumber between the symmetric and antisymmetric stretching modes of the carboxylate group) than non-bonded ionic carboxylates, while a bidentate chelating bond will lead to a smaller $\Delta$. Furthermore, the fundamental causes of the changes in $\Delta$ have been demonstrated to be primarily due to the carboxylate bond lengths and angles. It has been seen that almost the entirety of the effect of a bidentate bond comes from how the bond with the metal containing species reduces the carboxylate bond angle. A significant proportion of the effect of a monodentate bond comes from how the metal containing species affects the carboxylate bond lengths. The main cause of the shift in wavenumbers is geometry, i.e. changing bond length results in larger delta while decreasing bond angle results in slightly decreased delta. These changes in geometry are related to the bonding configuration; monodentate lengthens one bond and shortens the other, while bidentate reduces the bond angle. The partial charges of the atoms in the carboxylate group also affect the wavenumbers of the vibrational modes. The analysis herein will aid understanding of future experimental results and provides confidence in the use of the guidelines for aqueous systems.

\section{Computational Methods}

First, the carboxylate bond angles and lengths are specified to mimic bidentate and monodentate bonding, then metal cation-carboxylate anion systems were investigated; the wavenumbers of their vibrations are examined to understand the influence of the carboxylate geometry on the IR spectra. In Sutton et al. $2015^{[14]}$ it was shown that using the M05$2 X /$ cc-pVTZ level of theory with the SMD solvent model ${ }^{[15]}$ for modelling the vibrational wavenumbers of free carboxylate anions in aqueous solution a mean unsigned error of $4 \mathrm{~cm}^{-1}$ for $v_{\mathrm{a}}$ and $28 \mathrm{~cm}^{-1}$ for $v_{\mathrm{s}}$ was obtained. This model chemistry was originally proposed for modelling carboxylic acids in Sutton et al. $2012^{[16]}$ by determining the most accurate methods to calculate the $\mathrm{p} K_{\mathrm{a}}$. This model chemistry is utilized in this investigation. All calculations are performed using Gaussian 09. [17] Further details of the computational method are detailed in the Supporting Information. 


\section{Acknowledgements}

This work is supported by the Australian Research Council (ARC) through Discovery Project DP098570 (GVF). CCRS is the recipient of an APA scholarship. GdS is supported by the ARC Future Fellowship (FT130101304) and Discovery Project (DP110103889 and DP130100862) programs.

Keywords: IR spectroscopy • Carboxylate ligands • Chelates • Carboxylic acids $\cdot$ Computational chemistry

[1] G. B. Deacon, R. J. Phillips, Coordination Chemistry Reviews 1980, 33, 227.

[2] K. Nakamoto, Infrared and Raman spectra of inorganic and coordination compounds / Kazuo Nakamoto, Hoboken, N.J. : Wiley, 6th ed., 2009.

[3] a) P. C. Hidber, T. J. Graule, L. J. Gauckler, Journal of the American Ceramic Society 1996, 79, 1857; b) S. M. Barlow, K. J. Kitching, S. Haq N. V. Richardson, Surface Science 1998, 401, 322; c) M. Matzapetakis, C. P. Raptopoulou, A. Terzis, A. Lakatos, T. Kiss, A. Salifoglou, Inorganic Chemistry 1999, 38, 618; d) N. J. Nicholas, G. V. Franks, W. A. Ducker, Langmuir 2012, 28, 7189; e) J. E. Tackett, Applied Spectroscopy 1989, 43, 483; f) A. Barth, C. Zscherp, Quarterly Reviews of Biophysics 2002, 35, 369; g) S. Sakohara, M. Ishida, M. A. Anderson, Journal of Physical Chemistry B 1998, 102, 10169; h) M. Matzapetakis, N. Karligiano, A. Bino, M. Dakanali, C. P. Raptopoulou, V. Tangoulis, A Terzis, J. Giapintzakis, A. Salifoglou, Inorganic Chemistry 2000, 39, 4044; i) M. Matzapetakis, M. Kourgiantakis, M. Dakanali, C. P Raptopoulou, A. Terzis, A. Lakatos, T. Kiss, I. Banyai, L. Iordanidis, T. Mavromoustakos, A. Salifoglou, Inorganic Chemistry 2001, 40, 1734; j) S. J. Hug, D. Bahnemann, Journal of Electron Spectroscopy and Related Phenomena 2006, 150, 208; k) V. Zeleňák, Z. Vargová, K. Györyová, Spectrochimica Acta Part A: Molecular and Biomolecular Spectroscopy 2007, 66, 262; I) L. H. Do, T. Hayashi, P. Moenne-Loccoz, S. J. Lippard, Journal of the American Chemical Society 2010, 132, 1273 ; m) K. Kalyanasundaram, M. Gratzel, Coordination Chemistry Reviews 1998, 177, 347; n) G. Busca, V. Lorenzelli, Materials Chemistry 1982, 7, 89; o) C. J. Kepert, T. J. Prior, M. J. Rosseinsky Journal of the American Chemical Society 2000, 122, 5158; p)A . Barth, Progress in Biophysics \& Molecular Biology 2000, 74, 141; q) M. K. Nazeeruddin, R. Humphry-Baker, P. Liska, M. Gratzel, Journal of Physical Chemistry B 2003, 107, 8981; r) Z. S. Wang, Y. Cui, Y. DanOh, C. Kasada, A. Shinpo, K. Hara, Journal of Physical Chemistry C 2007, 111, 7224; s) H. N. Tian, X. C. Yang, R. K. Chen, R. Zhang, A. Hagfeldt, L. C. Sunt, Journal of Physical Chemistry C 2008, 112, 11023; t) R. Fandos, A. Otero, A. M. Rodriguez, S. Suizo, Journal of Organometallic Chemistry 2014, 759, 74; u) X. X. Wu, B. Ding, P. Yang, X. G. Wang, G. X. Du, Synthesis and Reactivity in Inorganic MetalOrganic and Nano-Metal Chemistry 2014, 44, 486; v) B. Yue, Y. N. Chen, H. B. Chu, Y. R. Qu, A. L. Wang, Y. L. Zhao, Inorganica Chimica Acta 2014, 414, 39

[4] a) F. Jones, J. B. Farrow, W. van Bronswijk, Langmuir 1998, 14, 6512; b) H. Wagata, N. Ohashi, T. Taniguchi, K. I. Katsumata, K. Okada, N.
Matsushita, Crystal Growth \& Design 2010, 10, 4968; c) A. Hagfeldt, G. Boschloo, L. C. Sun, L. Kloo, H. Pettersson, Chemical Reviews 2010 110, 6595; d) M. Tabatabaee, S. Amjad, S. Tabatabaei, K. Molcanov, Synthesis and Reactivity in Inorganic Metal-Organic and Nano-Metal Chemistry 2014, 44, 507; e) H. Sheykhi, E. Safaei, Journal of the Chinese Chemical Society 2014, 61, 471.

[5] a) A. Barth, Biochimica Et Biophysica Acta-Bioenergetics 2007, 1767 1073; b) R. J. Debus, Coordination Chemistry Reviews 2008, 252, 244.

[6] a) I. Turel, Coordination Chemistry Reviews 2002, 232, 27; b) L. Protesescu, M. Tudorache, S. Neatu, M. N. Grecu, E. Kemnitz, P. Filip, V. I. Parvulescu, S. M. Coman, Journal of Physical Chemistry C 2011, 115, 1112.

[7] a) A. Gericke, H. Huhnerfuss, Thin Solid Films 1994, 245, 74; b) D. L. Casher, L. Kobr, J. Michl, Langmuir 2012, 28, 1625; c) J. SimonKutscher, A. Gericke, H. Huhnerfuss, Langmuir 1996, 12, 1027; d) A. M. Jubb, W. Hua, H. C. Allen, Annual Review of Physical Chemistry, Vol 63 2012, 63, 107; e) I. V. Chernyshova, S. Ponnurangam, P. Somasundaran, Langmuir 2011, 27, 10007; f) W. F. Dong, R. M. Wang, G. Z. Mao, H. Mohwald, Soft Matter 2006, 2, 686.

[8] a) J. E. Tackett, Applied Spectroscopy 1989, 43, 490; b) S. M. VargasVasquez, L. B. Romero-Zeron, B. MacMillan, Chemical Engineering Communications 2010, 197, 491.

[9] S. Ardo, G. J. Meyer, Chemical Society Reviews 2009, 38, 115.

[10] a) J. Zhang, S. Ohara, M. Umetsu, T. Naka, Y. Hatakeyama, T. Adschiri, Advanced Materials 2007, 19, 203; b) T. A. Cheema, G. Garnweitner Crystengcomm 2014, 16, 3366.

[11] a) M. Nara, H. Torii, M. Tasumi, Journal of Physical Chemistry 1996, 100, 19812; b) L. J. Ma, Y. Li, L. Li, Y. Q. Wu, R. Buchet, Y. H. Ding, Spectrochimica Acta Part a-Molecular and Biomolecular Spectroscopy 2009, 72, 306; c) L. C. Bichara, M. V. F. Bimbi, C. A. Gervasi, P. E. Alvarez, S. A. Brandan, Journal of Molecular Structure 2012, 1008, 95; d) M. C. Bernini, J. C. Garro, E. V. Brusau, G. E. Narda, E. L. Varetti, Journal of Molecular Structure 2008, 888, 113 .

[12] a) A. Berna, J. M. Delgado, J. M. Orts, A. Rodes, J. M. Feliu, Electrochimica Acta 2008, 53, 2309; b) J. M. Delgado, R. Blanco, J. M. Orts, J. M. Perez, A. Rodes, Electrochimica Acta 2010, 55, 2055; c) J. M. Delgado, R. Blanco, J. M. Perez, J. M. Orts, A. Rodes, Journal of Physical Chemistry C 2010, 114, 12554

[13] a) M. Calatayud, S. E. Collins, M. A. Baltanas, A. L. Bonivardi, Physical Chemistry Chemical Physics 2009, 11, 1397; b) A. Lenz, L. Selegard, F. Soderlind, A. Larsson, P. O. Holtz, K. Uvdal, L. Ojamae, P. O. Kall, Journal of Physical Chemistry C 2009, 113, 17332.

[14] C. C. R. Sutton, G. V. Franks, G. da Silva, Spectrochimica Acta Part A: Molecular and Biomolecular Spectroscopy 2015, 134, 535.

[15] A. V. Marenich, C. J. Cramer, D. G. Truhlar, Journal of Physical Chemistry B 2009, 113, 6378.

[16] C. C. R. Sutton, G. V. Franks, G. da Silva, Journal of Physical Chemistry B 2012, 116, 11999.

[17] M. J. Frisch, G. W. Trucks, H. B. Schlegel et al.,, Gaussian, Inc. Wallingford CT, 2009.

[18] a) R. S. Mulliken, Journal of Chemical Physics 1955, 23, 1833; b) E. Lewars, Computational chemistry [electronic resource], $2^{\text {nd }}$ ed, Springer, Dordrecht, 2010, pp. 345-352. [3] ... 
Entry for the Table of Contents (Please choose one layout)

Layout 2:

\section{FULL PAPER}

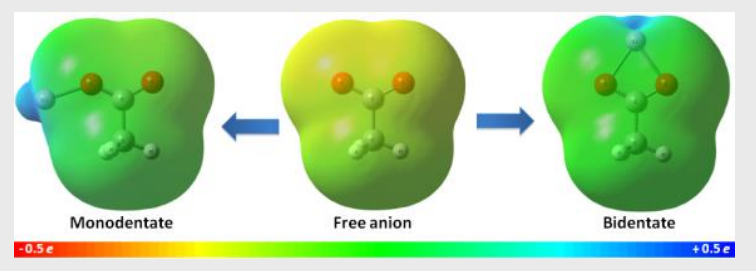

IR carboxylate stretching modes are widely used to infer if the geometry of the bonding is monodentate or bidentate. This belief was validated with ab-initio modelling for formate and acetate aqueous metal-carboxylate complexes. It was shown that these shifts in wavenumber occur primarily due to how bonding with the metal changes the carboxylate $\mathrm{C}-\mathrm{O}$ bond lengths and $\mathrm{O}-\mathrm{C}-\mathrm{O}$ angle.
Catherine. C. R. Sutton, Gabriel da Silva, and George V. Franks*

Page No. - Page No.

Modelling the IR spectra of aqueous metal carboxylate complexes: correlation between bonding geometry and stretching mode wavenumber shifts 